\title{
Changes in smoking cessation advice from GDPs
}

\author{
Smoking cessation interventions in the Oxford region: changes in dentists' attitudes and reported practices \\ 1996-2001 J. H. John, D. Thomas and D. Richards Br Dent J 2003; 195: 270-275
}

\section{Objectives}

To investigate dentists' current beliefs and practices (2001) with respect to smoking cessation interventions and any changes that might have occurred since the last survey in 1996.

Design

Postal questionnaire survey.

\section{Setting}

General dental practitioners on the health authority lists of Berkshire, Buckinghamshire, Oxfordshire and Northamptonshire.

Results

A response rate of 71\% (696/984) was achieved. The majority of dentists (88.6\%) thought that dentists should encourage their patients to stop smoking (increase of 6.3\% since 1996; 95\%CI 2.6, $10.2 ; \mathrm{p}=0.001$ ), although only $42.2 \%$ believed dentists were effective in this area (non-significant increase of 4.8\% since 1996). Only 48.4\% routinely recorded their patient's smoking status (increase of 30.3\% since 1996; 95\%CI 25.4, 34.9; p<0.001) and only $26.9 \%$ always discussed the habit with smokers (increase of 9.5\% since 1996; 95\%CI 5.1, 13.9; $\mathrm{p}<0.001)$. Less than $10 \%$ reported a good knowledge of NRT or Bupropion. About half of respondents requested more training and resources.

\section{Conclusions}

Dentists generally have positive attitudes to being involved in smoking cessation interventions, but although there are significant increases between 1996 and the current survey, few are active in this area. More training and resources may encourage dentists to be more proactive in helping their patients to stop smoking.

\section{IN BRIEF}

- Respondents generally believed that they should encourage their patients to stop smoking, and that oral health problems and oral cancer were major motivating factors in encouraging smokers to stop smoking.

- About a third more dentists routinely recorded their patients' smoking status in 2001 than in 1996 but the final proportion was still less than half of all dentists. Only a quarter always raised the subject with smokers.

- Less than half of respondents believed that dentists are effective in helping patients stop smoking although most believed that doctors were effective.

- Very few respondents reported a good knowledge of nicotine replacement therapy (NRT) and about half requested more training and resources.

\section{COMMENT}

Smoking is the single greatest threat to public health across the world. In the UK every day about 330 people die prematurely due to a smoking related disease. This is equivalent to the fatalities associated with a jumbo jet crashing every day and all the passengers on board being killed. Although rates of smoking in the UK have declined, tobacco use amongst socially deprived communities remains very high and is a significant contributor to health inequalities. In recognition of the magnitude of the problem, the Government's anti-smoking strategy is implementing a range of approaches to prevent smoking, particularly amongst young people and to support individuals who want to quit the addictive habit. An important element of the strategy is developing more effective and comprehensive smoking cessation services within the NHS. Challenging national targets have been set which will require coordinated action by health professionals, particularly those working in primary care settings.

Several studies have assessed dentists' attitudes and practices relating to smoking cessation, both in the UK and overseas. This study however provides a unique insight into changes in dentists' beliefs and practices over a five year period during which a great deal of public health activity has focused on smoking. Overall the results of this study are very encouraging and provide some positive indication of greater involvement of dentists in smoking cessation activities. However although a very high proportion of dentists questioned in this study reported positive beliefs towards smoking cessation, the proportion who are actively engaged in routine smoking advice is still relatively low. Less than $50 \%$ routinely record patients' smoking status and just over a quarter reported to always discuss smoking with their patients. Very few of the study sample had a good knowledge of nicotine replacement therapies or Bupropion, two very important elements of effective clinical cessation support. An encouraging finding was that amongst younger dentists a higher proportion were more involved in cessation activities.

The results of this study confirm previous findings that despite positive beliefs, many dentists still are not actively engaged in smoking cessation. The challenge is translating dentists' beliefs into sustained changes in their clinical activity. To achieve this requires more than just simple education and training. The perceived and real barriers that are preventing a change in practice need to be addressed. The conditions in which practitioners operate should facilitate their involvement in this area of clinical prevention. A range of appropriate incentives need to be identified. Team working both within the dental setting and beyond are critical elements for success. For example, across the country specialist cessation services have been established to support smokers who require more detailed support to quit smoking. Dental practitioners have an important role in referring any patients who may need this assistance. Tailored training and skills development for the dental team are also required. These training initiatives need to engage practitioners by highlighting the importance and relevance of smoking cessation to dentistry. University College London 